TAIWANESE JOURNAL OF MATHEMATICS

Vol. 11, No. 1, pp. 239-253, March 2007

This paper is available online at http://www.math.nthu.edu.tw/tjm/

\title{
CONVERGENCE CRITERION AND CONVERGENCE BALL OF THE KING-WERNER METHOD UNDER THE RADIUS LIPSCHITZ CONDITION
}

\author{
Xintao Ye, Chong Li and Liying Hou
}

\begin{abstract}
The convergence of the King-Werner method for finding zeros of nonlinear operators is analyzed. Under the hypothesis that the derivative of $f$ satisfies the radius Lipschitz condition with $L$-average, the convergence criterion and the convergence ball for the King-Werner method are given. Applying the results to some particular functions $L(u)$, we get the convergence theorems in [7] and [1] as well as some new results.
\end{abstract}

\section{INTRODUCTION}

Let $E$ and $F$ be real or complex Banach spaces and let $f$ be a nonlinear operator from a nonempty subset $U$ of $E$ to $F$. Solving the operator equation $f(x)=$ 0 is a basic and important problem in applied and computational mathematics. The most common methods to solve the equation are the Newton method and its variations. One of the variations is the well-known King-Werner method, which was independently proposed and studied by King [3] and Werner [14]. The King-Werner method with initial points $x_{0}=y_{0}$ is defined by

$$
\begin{aligned}
x_{n+1} & =x_{n}-f^{\prime}\left(y_{n}\right)^{-1} f\left(x_{n}\right), \\
z_{n+1} & =x_{n+1}-f^{\prime}\left(y_{n}\right)^{-1} f\left(x_{n+1}\right), \quad n=0,1, \cdots . \\
y_{n+1} & =\left(x_{n+1}+z_{n+1}\right) / 2 .
\end{aligned}
$$

The number of the evaluations of the function value in the King-Werner method is similar to that in the Newton method but its convergence order is raised to $1+\sqrt{2}$.

Received January 29, 2005, accepted October 26, 2005.

Communicated by Tien-Yien Li.

2000 Mathematics Subject Classification: 65H10.

Key words and phrases: King-Werner method, Nonlinear operator equation, Convergence ball.

This work was supported in part by the National Natural Science Foundation of China (Grant No.10271025). 
Hence, the King-Werner method is more efficient in practice and more preferred. In [1], Han and Wang gave the convergence criterion for the method under the weak condition introduced in [11]. Recently, Wang, Li and Lai established in [7] a unified convergence theorem for the class of operators whose second derivatives satisfy the Lipschiz condition. In the spirit of Wang's idea in [9] and [10], we will introduce, in the present paper, the notion of the radius Lipschitz condition with $L$-average and then study the convergence of the King-Werner method. More precisely, under the hypothesis that $f^{\prime}\left(x_{0}\right)^{-1} f^{\prime \prime}$ satisfies the radius Lipschitz condition with $L$-average around the initial point, a convergence criterion is established in Section 3. Another interesting question for an iteration method is to determine its convergence ball. A ball $\mathbf{B}\left(x^{*}, r\right) \subset E$ with center $x^{*}$ and radius $r$ is called a convergence ball of an iteration method if the sequence generated by the method starting from any point in it converges to $x^{*}$. The radii of convergence balls for the Newton method, were estimated in [4-6, 9, 12], and for Chebyshev-Halley methods in [2, 13]. In Section 4, the radii of convergence balls of the King-Werner method are estimated under the hypothesis that $f^{\prime}\left(x_{0}\right)^{-1} f^{\prime \prime}$ satisfies the radius Lipschitz condition with $L$-average around the solution. Finally, applications are provided in final section, where we extend some known results.

The result on the convergence ball in the present paper is new even in the special cases when $f^{\prime}\left(x_{0}\right)^{-1} f^{\prime \prime}$ satisfies classical Lipschitz condition or when $f$ is analytic.

\section{PReLIMINARIES}

Let $E$ and $F$ be real or complex Banach spaces. For $x_{0} \in E$ and $r>0$, let $\mathbf{B}\left(x_{0}, r\right)$ and $\overline{\mathbf{B}\left(x_{0}, r\right)}$ denote the open and closed ball with center $x_{0}$ and radius $r$, respectively. Let $f: \overline{\mathbf{B}\left(x_{0}, r\right)} \rightarrow F$ be a nonlinear operator with continuous Fréchet derivative $f^{\prime}$. We assume that the inverse $f^{\prime}\left(x_{0}\right)^{-1}$ of $f^{\prime}\left(x_{0}\right)$ exists. In order to study the convergence of the King-Werner method, we introduce the following notion and some related properties. Throughout the whole paper, let $L$ be a function on the interval $[0, R]$ with $L(0)>0$ such that its derivative $L^{\prime}$ is positive and nondecreasing. Here, $R$ is some positive number such that $\int_{0}^{R}(R-u) L(u) \mathrm{d} u \geq R$.

Definition 2.1. $f^{\prime}\left(x_{0}\right)^{-1} f^{\prime \prime}(x)$ is said to satisfy the radius Lipschitz condition with $L$-average on $\mathbf{B}\left(x_{0}, r\right)$ if

$$
\begin{gathered}
\left\|f^{\prime}\left(x_{0}\right)^{-1}\left(f^{\prime \prime}\left(x^{\prime}\right)-f^{\prime \prime}(x)\right)\right\| \leq L\left(\left\|x-x_{0}\right\|+\left\|x^{\prime}-x\right\|\right)-L\left(\left\|x-x_{0}\right\|\right), \\
\forall x \in \overline{\mathbf{B}\left(x_{0}, r\right)}, \quad \forall x^{\prime} \in \overline{\mathbf{B}\left(x, r-\left\|x-x_{0}\right\|\right)} .
\end{gathered}
$$


Let $r>0$ be such that

$$
\int_{0}^{r} L(u) \mathrm{d} u=1
$$

and let

$$
b=\int_{0}^{r} u L(u) \mathrm{d} u
$$

For a constant $\beta \in(0, b]$, we define the majorizing function as follows.

$$
h(t)=\beta-t+\int_{0}^{t}(t-u) L(u) \mathrm{d} u, \quad \forall t \in[0, R] .
$$

Then

$$
\begin{gathered}
h(0)=\beta>0, \quad h(\beta)>0, \quad h(r)=\beta-b \leq 0, \quad h(R) \geq \beta>0, \\
h^{\prime}(t)<0, \quad h^{\prime \prime}(t)=L(t) \geq 0, \quad \forall t \in(0, r] .
\end{gathered}
$$

Hence, the following Lemma holds.

Lemma 2.1. If $\beta \leq b$, the function $h$ has two zeros in $[0, R]$, which are denoted by $t^{*}$ and $t^{* *}$, respectively. They satisfy

$$
\beta<t^{*}<r<t^{* *}<R
$$

if $\beta<b$, and $t^{*}=t^{* *}$ if $\beta=b$.

Let $\left\{t_{n}\right\},\left\{s_{n}\right\}$ and $\left\{r_{n}\right\}$ denote respectively the corresponding sequences generated by the King-Werner method for the majorizing function $h$ with initial points $t_{0}=s_{0}=0$, that is,

$$
\begin{aligned}
t_{n+1} & =t_{n}-h^{\prime}\left(s_{n}\right)^{-1} h\left(t_{n}\right), \\
r_{n+1} & =t_{n+1}-h^{\prime}\left(s_{n}\right)^{-1} h\left(t_{n+1}\right), \\
s_{n+1} & =\left(t_{n+1}+r_{n+1}\right) / 2 .
\end{aligned}
$$

The basic technique of establishing the convergence of the King-Werner method is to show the iterative sequences generated by (1.1) for an operator $f$ are majorized by the corresponding ones for the majorizing function $h$. Hence we need to establish the convergence properties of these sequences for $h$, which are described in the following lemma. 
Lemma 2.2. Let $\left\{t_{n}\right\},\left\{s_{n}\right\},\left\{r_{n}\right\}$ be defined by (2.6) and let $t^{*} \in(0, r]$ be the smaller positive zero of $h(t)=0$. Then $\left\{t_{n}\right\},\left\{s_{n}\right\},\left\{r_{n}\right\}$ converge to $t^{*}$ increasingly and monotonically. Moreover, they satisfy the following inequalities:

$$
0=t_{0}=s_{0} \leq t_{n} \leq s_{n} \leq r_{n} \leq t_{n+1} \leq t^{*} \leq r, \quad n=0,1,2, \ldots
$$

Proof. It suffices to show (2.7). We will prove the conclusion by the mathematical induction. Clearly, (2.7) is true for $n=1$. Now assume (2.7) is true for $n=k$, that is,

$$
0=t_{0}=s_{0} \leq t_{k} \leq s_{k} \leq r_{k} \leq t_{k+1} \leq t^{*} \leq r .
$$

Then, from (2.4), (2.5) and (2.6), we have

$$
r_{k+1}-t_{k+1}=-h^{\prime}\left(s_{k}\right)^{-1} h\left(t_{k+1}\right)>0
$$

and

$$
r_{k+1}=t_{k+1}-h^{\prime}\left(s_{k}\right)^{-1} h\left(t_{k+1}\right) \leq t_{k+1}-h^{\prime}\left(t_{k+1}\right)^{-1} h\left(t_{k+1}\right) \leq t^{*} .
$$

Note that $s_{k+1}$ is the midpoint of $t_{k+1}$ and $r_{k+1}$. By (2.8), (2.9) and (2.10), we get

$$
s_{k} \leq t_{k+1} \leq s_{k+1} \leq r_{k+1} \leq t^{*} .
$$

On the other hand, since $h\left(t_{k+1}\right)>0$ and $h^{\prime}(t)^{-1}$ is monotonically decreasing on $(0, r)$, it follows from (2.11) that

$$
t_{k+2}-r_{k+1}=-h\left(t_{k+1}\right)\left[h^{\prime}\left(s_{k+1}\right)^{-1}-h^{\prime}\left(s_{k}\right)^{-1}\right]>0 .
$$

In addition, similar to the proof of (2.10), one has $t_{k+1} \leq t^{*}$. Therefore, (2.7) holds for $n=k+1$. The proof is completed.

The following lemma whose proof is similar to that of [8, Lemma 2.5] will be useful in the next section.

\section{Lemma 2.3. Suppose that}

$$
\left\|f^{\prime}\left(x_{0}\right)^{-1} f^{\prime \prime}\left(x_{0}\right)\right\| \leq L(0)
$$

and $f^{\prime}\left(x_{0}\right)^{-1} f^{\prime \prime}(x)$ satisfies the radius Lipschitz condition with L-average on $\mathbf{B}\left(x_{0}, r\right)$. Then, for each $x \in \mathbf{B}\left(x_{0}, r\right)$,

(i) $\left\|f^{\prime}\left(x_{0}\right)^{-1} f^{\prime \prime}(x)\right\| \leq h^{\prime \prime}\left(\left\|x-x_{0}\right\|\right)$,

(ii) $f^{\prime}(x)^{-1}$ exists and

$$
\left\|f^{\prime}(x)^{-1} f^{\prime}\left(x_{0}\right)\right\| \leq-h^{\prime}\left(\left\|x-x_{0}\right\|\right)^{-1} .
$$




\section{Convergence Criterion of the King-Werner Method}

Theorem 3.1. Suppose that $\left\|f^{\prime}\left(x_{0}\right)^{-1} f^{\prime \prime}\left(x_{0}\right)\right\| \leq L(0)$ and $f^{\prime}\left(x_{0}\right)^{-1} f^{\prime \prime}(x)$ satisfies the radius Lipschitz condition with L-average on $\mathbf{B}\left(x_{0}, r\right)$. Let $\beta=$ $\left\|f^{\prime}\left(x_{0}\right)^{-1} f\left(x_{0}\right)\right\|$. Then, if $\beta \leq b$, the King-Werner sequence $\left\{x_{n}\right\}$ generated by (1.1) converges to the solution of the equation $f(x)=0$ in $\mathbf{B}\left(x_{0}, r\right)$.

Proof. Let $\left\{t_{n}\right\},\left\{s_{n}\right\}$ and $\left\{r_{n}\right\}$ be the sequence defined by (2.6). By Lemma 2.2, the sequences $\left\{t_{n}\right\},\left\{s_{n}\right\},\left\{r_{n}\right\}$ converge increasingly and monotonically. Thus, to prove the convergence of the sequence $\left\{x_{n}\right\}$, it suffices to show the following inequalities:

$$
\left\|x_{n+1}-x_{n}\right\| \leq t_{n+1}-t_{n}, \quad n=0,1,2 \ldots
$$

For this end, we need only to prove the following inequalities:

$$
\begin{gathered}
\left\|z_{n}-x_{n}\right\| \leq r_{n}-t_{n}, \quad n=0,1, \ldots, \\
\left\|x_{n+1}-z_{n}\right\| \leq t_{n+1}-r_{n}, \quad n=0,1, \ldots
\end{gathered}
$$

Obviously, (3.1) holds for $n=0$. Consequently,

$$
\left\|x_{1}-z_{0}\right\|=\left\|x_{1}-x_{0}\right\|=\beta=t_{1}-t_{0}=t_{1}-r_{0} .
$$

Hence (3.2) holds for $n=0$. We proceed by the mathematical induction. For this purpose, assume (3.1) and (3.2) hold for all $n \leq k$, that is,

$$
\begin{gathered}
\left\|z_{n}-x_{n}\right\| \leq r_{n}-t_{n}, n=0,1, \cdots, k, \\
\left\|x_{n+1}-z_{n}\right\| \leq t_{n+1}-r_{n}, n=0,1, \cdots, k .
\end{gathered}
$$

Then,

$$
\begin{array}{r}
\left\|y_{n}-x_{n}\right\| \leq s_{n}-t_{n}, n=0,1, \cdots, k, \\
\left\|y_{n}-z_{n}\right\| \leq r_{n}-s_{n}, n=0,1, \cdots, k .
\end{array}
$$

Furthermore, for each $n=0,1,2, \ldots, k$,

$$
\begin{aligned}
\left\|x_{n+1}-x_{0}\right\| & \leq\left\|x_{n+1}-z_{n}\right\|+\left\|z_{n}-x_{n}\right\|+\ldots+\left\|z_{0}-x_{0}\right\| \\
& \leq t_{n+1}-r_{n}+r_{n}-t_{n}+\ldots+r_{0}-t_{0} \\
& =t_{n+1} \leq t^{*} .
\end{aligned}
$$

Hence,

$$
\left\|z_{k}-x_{0}\right\| \leq\left\|z_{k}-x_{k}\right\|+\left\|x_{k}-x_{0}\right\| \leq r_{k}
$$


and

$$
\left\|y_{k}-x_{0}\right\|=\left\|\frac{1}{2}\left(x_{k}+z_{k}\right)-x_{0}\right\| \leq \frac{1}{2}\left(t_{k}+r_{k}\right)=s_{k}
$$

Combining the above two inequalities gives $x_{k+1} \in \overline{\mathbf{B}\left(x_{0}, t^{*}\right)}$ and $z_{k} \in \overline{\mathbf{B}\left(x_{0}, t^{*}\right)}$. Thus, by (1.1) and Lemma 2.3, we obtain

$$
\begin{aligned}
\left\|z_{k+1}-x_{k+1}\right\| & =\left\|f^{\prime}\left(y_{k}\right)^{-1} f\left(x_{k+1}\right)\right\| \\
& \leq\left\|f^{\prime}\left(y_{k}\right)^{-1} f^{\prime}\left(x_{0}\right)\right\| \cdot\left\|f^{\prime}\left(x_{0}\right)^{-1} f\left(x_{k+1}\right)\right\| \\
& \leq-h^{\prime}\left(s_{k}\right)^{-1}\left\|f^{\prime}\left(x_{0}\right)^{-1} f\left(x_{k+1}\right)\right\| .
\end{aligned}
$$

By [11], $f\left(x_{k+1}\right)$ can be expressed as

$$
\begin{aligned}
f\left(x_{k+1}\right)= & \int_{0}^{1} \int_{0}^{1} f^{\prime \prime}\left(z_{k}+\eta \tau\left(x_{k+1}-z_{k}\right)\right)\left[\tau\left(x_{k+1}-z_{k}\right)\right] \mathrm{d} \eta \mathrm{d} \tau\left(x_{k+1}-z_{k}\right) \\
& +\int_{0}^{1} f^{\prime \prime}\left(y_{k}+\tau\left(z_{k}-y_{k}\right)\right)\left(z_{k}-y_{k}\right) \mathrm{d} \tau\left(x_{k+1}-z_{k}\right) \\
& +\int_{0}^{1}\left\{f^{\prime \prime}\left(y_{k}+\tau\left(y_{k}-x_{k}\right)\right)-f^{\prime \prime}\left(y_{k}+\tau\left(x_{k}-y_{k}\right)\right)\right\}(1-\tau) \mathrm{d} \tau\left(x_{k}-y_{k}\right)^{2} .
\end{aligned}
$$

Hence

$$
\begin{aligned}
& \left\|f^{\prime}\left(x_{0}\right)^{-1} f\left(x_{k+1}\right)\right\| \\
\leq & \int_{0}^{1} \int_{0}^{1}\left\|f^{\prime}\left(x_{0}\right)^{-1} f^{\prime \prime}\left(z_{k}+\eta \tau\left(x_{k+1}-z_{k}\right)\right)\right\| \tau \| x_{k+1} \\
& -z_{k}\|\mathrm{~d} \eta \mathrm{d} \tau\| x_{k+1}-z_{k} \| \\
& +\int_{0}^{1}\left\|f^{\prime}\left(x_{0}\right)^{-1} f^{\prime \prime}\left(y_{k}+\tau\left(z_{k}-y_{k}\right)\right)\right\|\left\|z_{k}-y_{k}\right\| \mathrm{d} \tau\left\|x_{k+1}-z_{k}\right\| \\
& +\int_{0}^{1} \| f^{\prime}\left(x_{0}\right)^{-1}\left(f^{\prime \prime}\left(y_{k}+\tau\left(y_{k}-x_{k}\right)\right)\right. \\
& \left.-f^{\prime \prime}\left(y_{k}+\tau\left(x_{k}-y_{k}\right)\right)\right)\|(1-\tau) \mathrm{d} \tau\| x_{k}-y_{k} \|^{2} .
\end{aligned}
$$

This together with Lemma 2.3 yields

$$
\begin{aligned}
& \left\|f^{\prime}\left(x_{0}\right)^{-1} f\left(x_{k+1}\right)\right\| \\
\leq & \int_{0}^{1} \int_{0}^{1} h^{\prime \prime}\left(\left\|z_{k}-x_{0}+\eta \tau\left(x_{k+1}-z_{k}\right)\right\|\right) \tau\left\|x_{k+1}-z_{k}\right\| \mathrm{d} \eta \mathrm{d} \tau\left\|x_{k+1}-z_{k}\right\|
\end{aligned}
$$




$$
\begin{aligned}
& +\int_{0}^{1} h^{\prime \prime}\left(\left\|y_{k}-x_{0}+\tau\left(z_{k}-y_{k}\right)\right\|\right)\left\|z_{k}-y_{k}\right\| \mathrm{d} \tau\left\|x_{k+1}-z_{k}\right\| \\
& +\int_{0}^{1}\left\{h^{\prime \prime}\left(\left\|y_{k}-x_{0}+\tau\left(x_{k}-y_{k}\right)\right\|+2 \tau\left\|y_{k}-x_{k}\right\|\right)\right. \\
& \left.-h^{\prime \prime}\left(\left\|y_{k}-x_{0}+\tau\left(x_{k}-y_{k}\right)\right\|\right)\right\}(1-\tau) \mathrm{d} \tau\left\|x_{k}-y_{k}\right\|^{2} .
\end{aligned}
$$

By (3.3)-(3.8), we obtain, for any $0 \leq \tau \leq 1$ and $0 \leq \eta \leq 1$,

$$
\begin{gathered}
\left\|z_{k}-x_{0}+\eta \tau\left(x_{k+1}-z_{k}\right)\right\| \leq r_{k}+\eta \tau\left(t_{k+1}-r_{k}\right), \\
\left\|y_{k}-x_{0}+\tau\left(z_{k}-y_{k}\right)\right\| \leq s_{k}+\tau\left(r_{k}-s_{k}\right), \\
\left\|\left(y_{k}-x_{0}\right)+\tau\left(x_{k}-y_{k}\right)\right\|+2 \tau\left\|y_{k}-x_{k}\right\| \leq s_{k}+\tau\left(r_{k}-s_{k}\right)
\end{gathered}
$$

and

$$
\left\|\left(y_{k}-x_{0}\right)+\tau\left(x_{k}-y_{k}\right)\right\| \leq s_{k}+\tau\left(t_{k}-s_{k}\right) .
$$

Consequently, by (3.6) and (3.11),

$$
\begin{aligned}
& \left\|f^{\prime}\left(x_{0}\right)^{-1} f\left(x_{k+1}\right)\right\| \\
\leq & \int_{0}^{1} \int_{0}^{1} h^{\prime \prime}\left(r_{k}+\eta \tau\left(t_{k+1}-r_{k}\right)\right)\left[\tau\left(t_{k+1}-r_{k}\right)\right] \mathrm{d} \eta \mathrm{d} \tau\left(t_{k+1}-r_{k}\right) \\
& +\int_{0}^{1} h^{\prime \prime}\left(s_{k}+\tau\left(r_{k}-s_{k}\right)\right)\left(r_{k}-s_{k}\right) \mathrm{d} \tau\left(t_{k+1}-r_{k}\right) \\
& +\int_{0}^{1}\left\{h^{\prime \prime}\left(s_{k}+\tau\left(r_{k}-s_{k}\right)\right)-h^{\prime \prime}\left(s_{k}+\tau\left(t_{k}-s_{k}\right)\right)(1-\tau)\right\} \mathrm{d} \tau\left(s_{k}-t_{k}\right)^{2} \\
= & h\left(t_{k+1}\right) .
\end{aligned}
$$

Combining this with (3.9) gives

$$
\left\|z_{k+1}-x_{k+1}\right\| \leq-h^{\prime}\left(s_{k}\right)^{-1} h\left(t_{k+1}\right)=r_{k+1}-t_{k+1},
$$

i.,e., (3.1) holds for $n=k+1$. Moreover, by (3.3), (3.4) and (3.13), one has, for any $0 \leq \tau \leq 1$,

$$
\left\|x_{k+1}-x_{k}\right\| \leq t_{k+1}-t_{k} \quad \text { and } \quad\left\|z_{k+1}-z_{k}\right\| \leq r_{k+1}-r_{k} .
$$

This implies

$$
\left\|y_{k+1}-y_{k}\right\| \leq s_{k+1}-s_{k} .
$$

Hence by (3.8)

$$
\left\|y_{k}-x_{0}+\tau\left(y_{k+1}-y_{k}\right)\right\| \leq s_{k}+\tau\left(s_{k+1}-s_{k}\right) .
$$


It follows from Lemma 2.3 that

$$
\begin{aligned}
& \left\|f^{\prime}\left(x_{0}\right)^{-1}\left(f^{\prime}\left(y_{k+1}\right)-f^{\prime}\left(y_{k}\right)\right)\right\| \\
\leq & \int_{0}^{1} \| f^{\prime}\left(x_{0}\right)^{-1}\left(f^{\prime \prime}\left(y_{k}+\tau\left(y_{k+1}-y_{k}\right)\right)\|\mathrm{d} \tau\| y_{k+1}-y_{k} \|\right. \\
\leq & \int_{0}^{1} h^{\prime \prime}\left(s_{k}+\tau\left(s_{k+1}-s_{k}\right)\right) \mathrm{d} \tau\left(s_{k+1}-s_{k}\right) \\
= & h^{\prime}\left(s_{k+1}\right)-h^{\prime}\left(s_{k}\right) .
\end{aligned}
$$

By (3.14), $\left\|y_{k+1}-x_{0}\right\| \leq t^{*} \leq r$. Thus, by Lemma 2.3 and (3.12), we have

$$
\begin{aligned}
& \left\|f^{\prime}\left(y_{k+1}\right)^{-1} f\left(x_{k+1}\right)\right\| \\
\leq & \left\|f^{\prime}\left(y_{k+1}\right)^{-1} f^{\prime}\left(x_{0}\right)\right\| \cdot\left\|f^{\prime}\left(x_{0}\right)^{-1} f\left(x_{k+1}\right)\right\| \leq-h^{\prime}\left(s_{k+1}\right)^{-1} h\left(t_{k+1}\right) .
\end{aligned}
$$

Therefore,

$$
\begin{aligned}
& \left\|x_{k+2}-z_{k+1}\right\| \\
= & \left\|\left(f^{\prime}\left(y_{k}\right)^{-1}-f^{\prime}\left(y_{k+1}\right)^{-1}\right) f\left(x_{k+1}\right)\right\| \\
= & \left\|f^{\prime}\left(y_{k}\right)^{-1}\left(f^{\prime}\left(y_{k+1}\right)-f^{\prime}\left(y_{k}\right)\right) f^{\prime}\left(y_{k+1}\right)^{-1} f\left(x_{k+1}\right)\right\| \\
\leq & \left\|f^{\prime}\left(y_{k}\right)^{-1} f^{\prime}\left(x_{0}\right)\right\| \cdot\left\|f^{\prime}\left(x_{0}\right)^{-1}\left(f^{\prime}\left(y_{k+1}\right)-f^{\prime}\left(y_{k}\right)\right)\right\| \cdot\left\|f^{\prime}\left(y_{k+1}\right)^{-1} f\left(x_{k+1}\right)\right\| \\
\leq & \left(-h^{\prime}\left(s_{k}\right)\right)^{-1}\left(h^{\prime}\left(s_{k+1}\right)-h^{\prime}\left(s_{k}\right)\right)\left(-h^{\prime}\left(s_{k+1}\right)^{-1} h\left(t_{k+1}\right)\right. \\
= & t_{k+2}-r_{k+1},
\end{aligned}
$$

i.e., (3.2) holds for $n=k+1$. This completes the proof of (3.1) and (3.2). The proof is completed.

\section{Convergence Ball of the King-werner Method}

Recall that $L(u)$ is a positive nondecreasing continuous function with $L(0)>0$ on the interval $[0, R]$ for some sufficiently large number $R>0$ and that $r$ satisfies that

$$
\int_{0}^{r} L(u) \mathrm{d} u=1
$$

By Lemma 2.3, we have

Lemma 4.1. Suppose that

$$
\left\|f^{\prime}\left(x^{*}\right)^{-1} f^{\prime \prime}\left(x^{*}\right)\right\| \leq L(0)
$$


and $f^{\prime}\left(x^{*}\right)^{-1} f^{\prime \prime}(x)$ satisfies the radius Lipschitz condition with L-average on $\mathbf{B}\left(x^{*}, r\right)$. Then, for each $x \in \mathbf{B}\left(x^{*}, r\right)$,

(i) $\left\|f^{\prime}\left(x^{*}\right)^{-1} f^{\prime \prime}(x)\right\| \leq L\left(\left\|x-x^{*}\right\|\right)$,

(ii) $f^{\prime}(x)^{-1}$ exists and

$$
\left\|f^{\prime}(x)^{-1} f^{\prime}\left(x^{*}\right)\right\| \leq \frac{1}{1-\int_{0}^{\left\|x-x^{*}\right\|} L(u) \mathrm{d} u} .
$$

Let

$$
p(t)=\frac{\int_{0}^{1}[u L(t u)+2(1-u) L(t(2 u+1))] t \mathrm{~d} u}{1-\int_{0}^{t} L(u) \mathrm{d} u} .
$$

Note that $p(t)$ increases monotonically from 0 to $+\infty$ when $t$ increases monotonically from 0 to $r$. Hence the following lemma holds.

Lemma 4.2. There exists a unique point $r_{0} \in(0, r)$ such that $p\left(r_{0}\right)=1$ and $p$ increases monotonically on $\left(0, r_{0}\right)$.

The main result of this section is stated as follows.

Theorem 4.1. Let $x^{*}$ be a solution of $f$. Suppose that

$$
\left\|f^{\prime}\left(x^{*}\right)^{-1} f^{\prime \prime}\left(x^{*}\right)\right\| \leq L(0)
$$

and that $f^{\prime}\left(x^{*}\right)^{-1} f^{\prime \prime}(x)$ satisfies the radius Lipschitz condition with L-average on $\mathbf{B}\left(x^{*}, r\right)$. Then, for any $x_{0} \in \mathbf{B}\left(x^{*}, r_{0}\right)$, the King-Werner sequence $\left\{x_{n}\right\}$ generated by (1.1) converges to $x^{*}$.

Proof. $\mathrm{n}+1$ Let $x_{0} \in \mathbf{B}\left(x^{*}, r_{0}\right)$ and let $q=p\left(\left\|x_{0}-x^{*}\right\|\right)<1$. It is sufficient to show that, for each $n=0,1,2, \ldots$,

$$
\left\|x_{n+1}-x^{*}\right\| \leq q\left\|x_{n}-x^{*}\right\|, \quad\left\|z_{n}-x^{*}\right\| \leq\left\|x_{n}-x^{*}\right\| .
$$

We will prove (4.3) by the mathematical induction. For this purpose, we need the following expression of $x_{n+1}-x^{*}$.

$$
\begin{aligned}
x_{n+1}-x^{*}= & f^{\prime}\left(\frac{x_{n}+z_{n}}{2}\right)^{-1} \int_{0}^{1} \int_{0}^{1} f^{\prime \prime}\left(x^{*}+\tau\left(x_{n}-x^{*}\right)\right. \\
& \left.+\eta\left(\frac{x_{n}+z_{n}}{2}-x^{*}-\tau\left(x_{n}-x^{*}\right)\right)\right) \\
& \cdot\left(\frac{x_{n}+z_{n}}{2}-x^{*}-\tau\left(x_{n}-x^{*}\right)\right) \mathrm{d} \tau \mathrm{d} \eta\left(x_{n}-x^{*}\right) .
\end{aligned}
$$


In fact,

$$
\begin{aligned}
x_{n+1}-x^{*}= & x_{n}-f^{\prime}\left(\frac{x_{n}+z_{n}}{2}\right)^{-1} f\left(x_{n}\right)-x^{*}+f^{\prime}\left(\frac{x_{n}+z_{n}}{2}\right)^{-1} f\left(x^{*}\right) \\
= & \left(x_{n}-x^{*}\right)-f^{\prime}\left(\frac{x_{n}+z_{n}}{2}\right)^{-1}\left[f\left(x_{n}\right)-f\left(x^{*}\right)\right] \\
= & \left(x_{n}-x^{*}\right)-f^{\prime}\left(\frac{x_{n}+z_{n}}{2}\right)^{-1} \int_{0}^{1} f^{\prime}\left(x^{*}+\tau\left(x_{n}-x^{*}\right)\right) \mathrm{d} \tau\left(x_{n}-x^{*}\right) \\
= & {\left[I-f^{\prime}\left(\frac{x_{n}+z_{n}}{2}\right)^{-1} \int_{0}^{1} f^{\prime}\left(x^{*}+\tau\left(x_{n}-x^{*}\right)\right) \mathrm{d} \tau\right]\left(x_{n}-x^{*}\right) } \\
= & f^{\prime}\left(\frac{x_{n}+z_{n}}{2}\right)^{-1}\left[f^{\prime}\left(\frac{x_{n}+z_{n}}{2}\right)-\int_{0}^{1} f^{\prime}\left(x^{*}+\tau\left(x_{n}-x^{*}\right)\right) \mathrm{d} \tau\right]\left(x_{n}-x^{*}\right) \\
= & f^{\prime}\left(\frac{x_{n}+z_{n}}{2}\right)^{-1} \int_{0}^{1} \int_{0}^{1} f^{\prime \prime}\left(x^{*}+\tau\left(x_{n}-x^{*}\right)\right. \\
& \left.+\eta\left(\frac{x_{n}+z_{n}}{2}-x^{*}-\tau\left(x_{n}-x^{*}\right)\right)\right) \\
& \cdot\left(\frac{x_{n}+z_{n}}{2}-x^{*}-\tau\left(x_{n}-x^{*}\right)\right) \mathrm{d} \tau \mathrm{d} \eta\left(x_{n}-x^{*}\right) .
\end{aligned}
$$

Therefore, (4.4) holds. Similarly, we also have

$$
\begin{aligned}
z_{n+1}-x^{*}= & f^{\prime}\left(\frac{x_{n}+z_{n}}{2}\right)^{-1} \int_{0}^{1} \int_{0}^{1} f^{\prime \prime}\left(x^{*}+\tau\left(x_{n+1}-x^{*}\right)\right. \\
& \left.+\eta\left(\frac{x_{n}+z_{n}}{2}-x^{*}-\tau\left(x_{n+1}-x^{*}\right)\right)\right) \\
& \cdot\left(\frac{x_{n}+z_{n}}{2}-x^{*}-\tau\left(x_{n+1}-x^{*}\right)\right) \mathrm{d} \tau \mathrm{d} \eta\left(x_{n+1}-x^{*}\right) .
\end{aligned}
$$

By (4.4),

$$
\begin{aligned}
x_{1}-x^{*}= & f^{\prime}\left(x_{0}\right)^{-1} \int_{0}^{1} \int_{0}^{1} f^{\prime \prime}\left(x^{*}+\tau\left(x_{0}-x^{*}\right)\right. \\
& \left.+\eta\left(x_{0}-x^{*}-\tau\left(x_{0}-x^{*}\right)\right)\right)(1-\tau) \mathrm{d} \tau \mathrm{d} \eta\left(x_{0}-x^{*}\right)^{2} .
\end{aligned}
$$

Write $t_{n}=\left\|x_{n}-x^{*}\right\|$. Then, it follows from Lemma 4.1 that

$$
\left\|x_{1}-x^{*}\right\| \leq \frac{1}{1-\int_{0}^{t_{0}} L(u) \mathrm{d} u} \int_{0}^{1} \int_{0}^{1} L\left(\tau t_{0}+\eta(1+\tau) t_{0}\right)(1+\tau) t_{0} \mathrm{~d} \tau \mathrm{d} \eta\left\|x_{0}-x^{*}\right\| .
$$


For each $t \in\left(0, r_{0}\right)$, simple calculation yields

$$
\begin{aligned}
& \int_{0}^{1} \int_{0}^{1} L(\tau t+\eta(1+\tau) t)(1+\tau) \mathrm{d} \tau \mathrm{d} \eta \\
= & \int_{0}^{1}\{u L(t u)+2(1-u) L[t(2 u+1)]\} \mathrm{d} u .
\end{aligned}
$$

Consequently,

$$
\left\|x_{1}-x^{*}\right\| \leq q\left\|x_{0}-x^{*}\right\|<\left\|x_{0}-x^{*}\right\| .
$$

By (1.1), $\left\|z_{0}-x^{*}\right\|=\left\|x_{0}-x^{*}\right\|$. Hence, (4.3) holds for $n=0$. Now assume that (4.3) holds for some $n$. Then, by (4.5), (4.6) and Lemma 4.1,

$$
\begin{aligned}
\left\|z_{n+1}-x^{*}\right\|= & \| f^{\prime}\left(\frac{x_{n}+z_{n}}{2}\right)^{-1} \int_{0}^{1} \int_{0}^{1} f^{\prime \prime}\left(x^{*}+\tau\left(x_{n+1}-x^{*}\right)\right. \\
& \left.+\eta\left(\frac{x_{n}+z_{n}}{2}-x^{*}-\tau\left(x_{n+1}-x^{*}\right)\right)\right) \\
& \cdot\left(\frac{x_{n}+z_{n}}{2}-x^{*}-\tau\left(x_{n+1}-x^{*}\right)\right) \mathrm{d} \tau \mathrm{d} \eta\left(x_{n+1}-x^{*}\right) \| \\
\leq & \frac{1}{1-\int_{0}^{t_{n}} L(u) \mathrm{d} u} \int_{0}^{1} \int_{0}^{1} L\left(\tau t_{n+1}+\eta\left(t_{n}+\tau t_{n+1}\right)\right) \\
& \left(t_{n}+\tau t_{n+1}\right) \mathrm{d} \tau \mathrm{d} \eta\left\|x_{n+1}-x^{*}\right\| \\
\leq & p\left(t_{n}\right)\left\|x_{n+1}-x^{*}\right\| \\
\leq & q\left\|x_{n+1}-x^{*}\right\| .
\end{aligned}
$$

On the other hand, by (4.4), (4.6) and Lemma 4.1,

$$
\begin{aligned}
& \left\|x_{n+2}-x^{*}\right\| \\
= & \| f^{\prime}\left(\frac{x_{n+1}+z_{n+1}}{2}\right)^{-1} \int_{0}^{1} \int_{0}^{1} f^{\prime \prime}\left(x^{*}\right. \\
& \left.+\tau\left(x_{n+1}-x^{*}\right)+\eta\left(\frac{x_{n+1}+z_{n+1}}{2}-x^{*}-\tau\left(x_{n+1}-x^{*}\right)\right)\right) \\
& \cdot\left(\frac{x_{n+1}+z_{n+1}}{2}-x^{*}-\tau\left(x_{n+1}-x^{*}\right)\right) \mathrm{d} \tau \mathrm{d} \eta\left(x_{n+1}-x^{*}\right) \| \\
\leq & \frac{1}{1-\int_{0}^{t_{n}} L(u) \mathrm{d} u} \int_{0}^{1} \int_{0}^{1} L\left(\tau t_{n+1}+\eta(1+\tau) t_{n+1}\right)(1+\tau) t_{n+1} \mathrm{~d} \tau \mathrm{d} \eta\left\|x_{n+1}-x^{*}\right\| \\
= & p\left(t_{n}\right)\left\|x_{n+1}-x^{*}\right\| \\
\leq & q\left\|x_{n+1}-x^{*}\right\| .
\end{aligned}
$$


This shows that (4.3) holds for $n+1$ and hence (4.3) holds for each $n$.

\section{Corollaries of Convergence Theorems}

In this section we will apply our main theorems to some special functions $L$ and then some results in [7] and [1] are recaptured.

Example 5.1. Given fixed positive constants $\gamma$ and $L>0$, take

$$
L(u)=\gamma+L u
$$

Then, by (2.2)and (2.3),

$$
r=\frac{2}{\gamma+\sqrt{\gamma^{2}+2 L}}, \quad b=\int_{0}^{r} u(\gamma+L u) \mathrm{d} u=\frac{2\left(\gamma+2 \sqrt{\gamma^{2}+2 L}\right)}{3\left(\gamma+\sqrt{\gamma^{2}+2 L}\right)^{2}} .
$$

Since

$$
p(t)=\frac{\frac{3}{2} t \gamma+2 t^{2} L}{1-\gamma t-\frac{1}{2} L t^{2}}
$$

the solution $r_{0}$ of the equation $p(t)=1$ on $(0, r)$ is

$$
r_{0}=\frac{-\gamma+\sqrt{\gamma^{2}+\frac{8}{5} L}}{2 L}
$$

Thus from Theorems 3.1 and 4.1, we immediately obtain the following corollaries. The first one was given in [7] while the second one is new.

Corollary 5.1. Suppose that $\left\|f^{\prime}\left(x_{0}\right)^{-1} f^{\prime \prime}\left(x_{0}\right)\right\| \leq \gamma$ and

$$
\begin{gathered}
\left\|f^{\prime}\left(x_{0}\right)^{-1}\left(f^{\prime \prime}\left(x^{\prime}\right)-f^{\prime \prime}(x)\right)\right\| \leq L\left\|x^{\prime}-x\right\|, \\
\forall x \in \overline{\mathbf{B}\left(x_{0}, r\right)}, \quad \forall x^{\prime} \in \overline{\mathbf{B}\left(x, r-\left\|x-x_{0}\right\|\right)} .
\end{gathered}
$$

Let $\beta=\left\|f^{\prime}\left(x_{0}\right)^{-1} f\left(x_{0}\right)\right\|$. Then, if $\beta \leq \frac{2\left(\gamma+2 \sqrt{\gamma^{2}+2 L}\right)}{3\left(\gamma+\sqrt{\gamma^{2}+2 L}\right)^{2}}$, the sequence $\left\{x_{n}\right\}$ generated by (1.1) with initial point $x_{0}$ converges to the solution $x^{*}$ of the equation $f(x)=0$ in $\mathbf{B}\left(x_{0}, r\right)$. and

Corollary 5.2. Let $x^{*}$ be a solution of $f$. Suppose that $\left\|f^{\prime}\left(x^{*}\right)^{-1} f^{\prime \prime}\left(x^{*}\right)\right\| \leq \gamma$

$$
\begin{gathered}
\left\|f^{\prime}\left(x^{*}\right)^{-1}\left(f^{\prime \prime}\left(x^{\prime}\right)-f^{\prime \prime}(x)\right)\right\| \leq L\left\|x^{\prime}-x\right\| \\
\forall x \in \mathbf{B}\left(x^{*}, r\right), \quad \forall x^{\prime} \in \mathbf{B}\left(x^{*}, r-\left\|x-x^{*}\right\|\right) .
\end{gathered}
$$


Let

$$
r_{0}=\frac{-\gamma+\sqrt{\gamma^{2}+\frac{8}{5} L}}{2 L} .
$$

Then for any $x_{0} \in \mathbf{B}\left(x^{*}, r_{0}\right)$, the sequence $\left\{x_{n}\right\}$ generated by (1.1) converges to the solution $x^{*}$ of the equation $f(x)=0$ in $\mathbf{B}\left(x^{*}, r\right)$.

Example 5.2. For fixed $\gamma>0$, let

$$
L(u)=\frac{2 \gamma}{(1-\gamma u)^{3}}
$$

Again, by (2.2) and (2.3),

$$
r=\left(1-\frac{1}{\sqrt{2}}\right) \frac{1}{\gamma}, \quad b=(3-2 \sqrt{2}) \frac{1}{\gamma} .
$$

Moreover,

$$
p(t)=\frac{3 \gamma t(1-\gamma t)}{(1-3 \gamma t)\left[2(1-\gamma t)^{2}-1\right]}
$$

and the solution $r_{0}$ of the equation $p(t)=1$ on $(0, r)$ is

$$
\begin{aligned}
r_{0}= & \frac{1}{36 \gamma}\{\sqrt[3]{809+18 \sqrt{3 \times 659} \mathrm{i}}(-1+\sqrt{3} \mathrm{i}) \\
& -\sqrt[3]{809-18 \sqrt{3 \times 659} \mathrm{i}}(1+\sqrt{3} \mathrm{i})+34\},
\end{aligned}
$$

where $\mathrm{i}=\sqrt{-1}$. From Theorems 3.1 and 4.1 again, we have the following corollaries.

Corollary 5.3. Suppose that $\left\|f^{\prime}\left(x_{0}\right)^{-1} f^{\prime \prime}\left(x_{0}\right)\right\| \leq 2 \gamma$, and

$$
\begin{gathered}
\left\|f^{\prime}\left(x_{0}\right)^{-1}\left(f^{\prime \prime}\left(x^{\prime}\right)-f^{\prime \prime}(x)\right)\right\| \leq \frac{2 \gamma}{\left(1-\gamma\left\|x-x_{0}\right\|-\gamma\left\|x^{\prime}-x\right\|\right)^{3}}-\frac{2 \gamma}{\left(1-\gamma\left\|x-x_{0}\right\|\right)^{3}}, \\
\forall x \in \overline{\mathbf{B}\left(x_{0},\left(1-\frac{1}{\sqrt{2}}\right) / \gamma\right)}, \quad \forall x^{\prime} \in \overline{\mathbf{B}\left(x,\left(1-\frac{1}{\sqrt{2}}\right) / \gamma-\left\|x-x_{0}\right\|\right)} .
\end{gathered}
$$

Let $\beta=\left\|f^{\prime}\left(x_{0}\right)^{-1} f\left(x_{0}\right)\right\|$. Then, if $\beta \leq(3-2 \sqrt{2}) \frac{1}{\gamma}$, the sequence $\left\{x_{n}\right\}$ generated by (1.1) with the initial point $x_{0}$ converges to the solution $x^{*}$ of the equation $f(x)=0$ in $\mathbf{B}\left(x_{0}, r\right)$.

Corollary 5.4. Let $x^{*}$ be a solution of $f$. Suppose that $\left\|f^{\prime}\left(x^{*}\right)^{-1} f^{\prime \prime}\left(x^{*}\right)\right\| \leq 2 \gamma$ and

$\left\|f^{\prime}\left(x^{*}\right)^{-1}\left(f^{\prime \prime}\left(x^{\prime}\right)-f^{\prime \prime}(x)\right)\right\| \leq \frac{2 \gamma}{\left(1-\gamma\left\|x-x^{*}\right\|+\gamma \| x^{\prime}-x^{*}\right)^{3}}-\frac{2 \gamma}{\left(1-\gamma\left\|x-x^{*}\right\|\right)^{3}}$ 


$$
\forall x \in \mathbf{B}\left(x^{*}, r\right), \quad \forall x^{\prime} \in \mathbf{B}\left(x^{*}, r-\left\|x-x^{*}\right\|\right) .
$$

Then for any $x_{0} \in \mathbf{B}\left(x^{*}, r_{0}\right)$, the sequence $\left\{x_{n}\right\}$ generated by (1.1) converges to the solution $x^{*}$ of the equation $f(x)=0$ in $\mathbf{B}\left(x^{*}, r_{0}\right)$.

Remark 5.1. Since the hypothesis stated in [1, Theorem 1] implies the one in Corollary 5.3 above, [1, Theorem 1] is a direct consequence of Corollary 5.3.

\section{ACKNOWLEDGMENT}

\section{REFERENCES}

1. D. F. Han and X. H. Wang, Convergence on a deformed Newton method, Applied Mathematics and Computation, 94 (1998), 65-72.

2. Z. D Huang, On a family of Chebyshev-Halley type methods in Banach space under weaker Smale condition, Numer. Math., JCU, 9 (2000), 37-43.

3. R. F. King, Tangent Methods for Nonlinear Equations, Numer. Math., 18 (1972), 298-304.

4. S. Smale, Newton's method estimates from data at one point, In: "The Merging of Disciplines: New Directions in Pure, Applied and Computational Mathematics", R. Ewing, K. Gross and C. Martin eds., New York, Spring, 1986, 185-196.

5. S. Smale, Complexity theory and numerical analysis, Acta Numer., 6 (1997), 523-551.

6. J. F. Traub and H. Wozniakowski, Convergence and complexity of Newton iteration, J. Assoc. Comput. Math., 29 (1979), 250-258.

7. X. H. Wang, C. Li and M. J. Lai, A unified convergence theory for Newton-Type methods for zeros of nolinear operators in Banach Spaces, BIT, 42 (2002), 206-213.

8. X. H. Wang and C. Li, On the united theory of the family of Euler-Halley type methods with cubical convergence in Banach Spaces, J. Comput. Math., 21 (2003), 195-200.

9. X. H. Wang, Convergence of Newton's method and uniqueness of the solution of equations in Banach Spaces, IMA Jounal of Numerical Analysis, 20 (2000), 123134.

10. X. H. Wang, Convergence of Newton's method and inverse function theorem in Banach Space, Mathematics of Computation, 225 (1999), 169-186.

11. X. H. Wang and D. F. Han, Criterion $\alpha$ and Newton's method in the weak condition, Math. Numer. sinica, 19 (1997), 103-112.

12. X. H. Wang, Convergent neighborhood on Newton's method, Kexue Tongbao (Chinese Science Bulletin), Special Issue of Math., Phy. and Chemistry, 25 (1980), 36-37. 
13. H. Y. Wang, C. Li and X. H. Wang, On the Relationship Between the Convergence Ball of the Euler Iteration in Banach Spaces and Its Dynamical Behavior on Riemann Spheres, Science in China(Ser. A), 46 (2003), 376-382.

14. W. Werner, Uber ein Verfahren der Ordnung $1+\sqrt{2}$ zur Nullstellenbestimmung, Numer. Math., 32 (1979), 333-342.

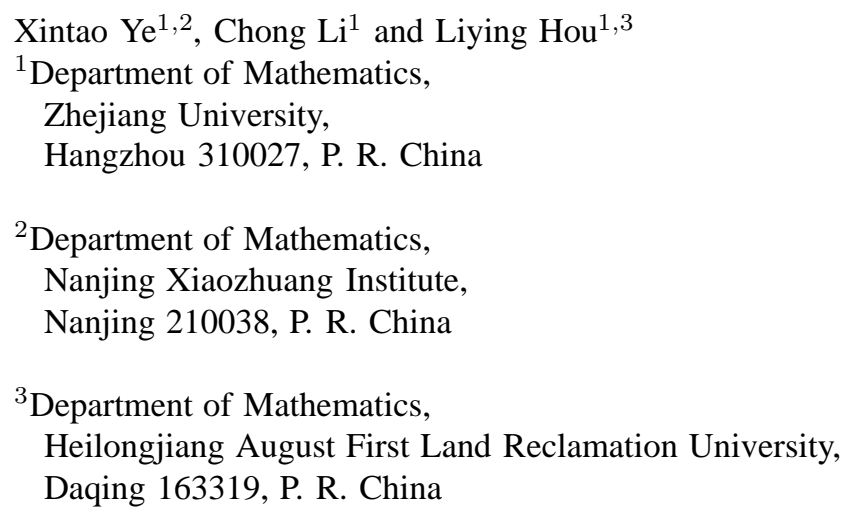

